Gereon Kopf

\title{
Zen, Erfahrung und Philosophie: Ueda Shizuteru und die Schöpfung einer Zen-Philosophie
}

\begin{abstract}
The philosophers of the so-called Kyoto school famously synthesized Zen Buddhist thought and the academic philosophy of 19th and early 20th century Europe in their work. Shizuteru Ueda, one of disciples of Nishitani, developed a model to categorize three different types of Zen discourse. However, in the English language literature "Zen" is frequently essentialized as "mystical" and as at odds with the philosophical project. Such a misconception not only does injustice to the diverse and vibrant traditions of Zen Buddhism, it also belittles the contributions many of their members have made to the philosophical discourse in general. This essay will introduce Ueda's model to present one of way of imagining "Zen philosophy" and to investigate how the project of philosophy in general can be understood from within the Japanese Zen Buddhist tradition. It will argue that since philosophy is not a matter of ideology but one of method, "doing philosophy within the Zen tradition" requires a translation of the Zen idiom. It will further demonstrate how Ueda identifies sources for and methods of philosophy within the Zen Buddhist traditions. The goal of this essay is to better understand the philosophical projects of one member of the Kyoto school, on the one side, and to envision a notion of "philosophy" that is relevant for the age of globalism and multiculturalism, on the other.
\end{abstract}

Keywords: Ueda Shizuteru, Zen, Philosophy, Nishitani Keiji, D. T. Suzuki, Hisamatsu Shin'ichi

DOI 10.1515/asia-2015-0023

\section{Einleitung: Zen vs. Philosophie?}

Dieser Artikel behandelt die philosophischen Fragen, die sich im Prozess der Bestimmung einer Zen-Philosophie ergeben. Um es gleich zu Beginn deutlich zu

Gereon Kopf, Professor of Religion, Department of Religion, Luther College, 700 College Dr. Decorah, IA 52101, USA. E-mail: kopfg@luther.edu

Übersetzung: Ralf Müller, Universität Hildesheim, Institut für Philosophie, Kulturwissenschaften und ästhetische Kommunikation. E-Mail: ralfmueller.eu@gmail.com 
sagen: Es ist nicht meine Absicht, „Philosophie“ oder „Zen“ zu definieren. Es soll auch nicht um die Frage gehen, ob eine Zen-Philosophie möglich ist, oder um den Versuch, Zen und Philosophie mit einander in Einklang zu bringen. Ein solcher Versuch beruht auf dem fundamentalen Kategorienfehler anzunehmen, Philosophie und Zen seien artverwandt, obwohl Philosophie eine akademische Disziplin oder Untersuchungsmethode bezeichnet und Zen eine religiös-philosophische Tradition; „Philosophie“ und „Zen“ wirklich definieren zu wollen, birgt die Gefahr, Zen und Philosophie als separate und ahistorische und somit unveränderliche Entitäten darzustellen.

Es besteht vielmehr die Notwendigkeit, die gegenwärtigen Diskurse innerhalb der Zen-Tradition $\mathrm{zu}$ durchdenken und $\mathrm{zu}$ klassifizieren, und zwar im Verhältnis zu den herkömmlichen Kategorien der akademischen Diskurse, wie sie zunächst durch den europäischen und dann nordamerikanischen Kontext bestimmt worden sind. Die Frage ist weniger, ob eine Zen-Philosophie möglich ist, sondern wie wir Diskurse kategorisieren und klassifizieren können, die sich so stark voneinander unterscheiden wie die „Dharma-Reden“ (j. seppō 説法) der Zen-Lehrer von der systematischen Philosophie Nishitani Keijis (1900-1990). Welche Kriterien ermöglichen eine solche Klassifikation? Liefert die Tradition des Zen-Buddhismus eigene Kriterien oder ist es notwendig, sich auf die Klassifikationssysteme der akademischen Philosophie zu stützen?

\section{Die ideologischen und politischen Fallen der Diskussion um „Zen-Philosophie“}

In seinem Buch Zen und die Welt (j. Zen to sekai 禅と世界) ${ }^{1}$ entwickelt Ueda Shizuteru (geboren 1926) ein heuristisches Modell, das nicht nur diese Fragen beantwortet, sondern darüber hinaus einen Weg weist, sowohl den Streit um Definitionen wie auch die oftmals in jenen Abhandlungen verborgene orientalisierende Rhetorik zu vermeiden, die „Zen“ gegen „Philosophie“ ausspielen.

\subsection{Die Kyoto-Schule und Zen}

Historisch betrachtet, stellt die Idee einer „Zen-Philosophie“ in Japan sowohl eine Möglichkeit wie auch ein Problem dar, als in der Meiji-Periode (1868-1912) die intellektuellen Traditionen Japans auf die euro-amerikanische Philosophie

1 Ueda 2002a: 11-181. 
treffen. Nishi Amane (1829-1897) führte in dieser Zeit den Unterschied zwischen japanischem „Denken“ (shisō) und westlicher „Philosophie“ (tetsugaku) ein. In gewisser Hinsicht spiegelt diese Terminologie zweierlei wider: zum einen die orientalisierende Unterscheidung zwischen „östlicher Weisheit“ und „westlichem Wissen“, zum anderen die japanische Aneignung dieser Unterscheidung in Form des Schlagworts „Japanischer Geist und westliche Technik“ (wakonyōsai). In beiden Fällen werden japanisches Denken und euro-amerikanische Philosophie als zwei grundsätzlich verschiedene Entitäten behandelt.

Die Philosophen der Kyoto-Schule betrachteten sich selbst im Besonderen und die japanische Kultur im Allgemeinen als Brücke zwischen den beiden kulturellen Hemisphären bzw. den darin gespiegelten Denkweisen. In diesem Kontext „entdeckte“ Watsuji Tetsurō (1889-1960) in den 1920er Jahren die Philosophie des Zen-Meisters Dōgen (1200-1253) für die akademische Welt. ${ }^{2}$ Watsuji war ein einflussreicher japanischer Philosoph, der eine eher randständige Rolle im Kreis der Kyoto-Schule spielte. Die Repräsentanten dieser Schule erklärten ihre Philosophie entweder als beeinflusst durch oder als Reflexion von Zen. Einige der Hauptrepräsentanten der Kyoto-Schule behaupteten, es bestehe eine Affinität zwischen buddhistischer Philosophie und ihrem eigenen Werk, manche stellten die Philosophie der Kyoto-Schule gar in Begriffen der buddhistischen Philosophie dar.

Nishida Kitarō (1870-1945) zum Beispiel vergleicht seine Philosophie an mehreren Stellen mit buddhistischen Texten ${ }^{3}$, buddhistischen Schulen, vor allem mit dem Buddhismus des Huayan (j. Kegon 華嚴) und des Tiantai (j. Tendai 天台) ${ }^{4}$, oder mit ,buddhistischer Philosophie“ (bukkyō tetsugaku 仏教哲学) im eigentlichen Sinne ${ }^{5}$. Letzeres verdient, eigens erwähnt zu werden, da es, obwohl nicht unmittelbar auf die Zen-Tradition anwendbar, Nishidas Offenheit zum Ausdruck bringt, den Terminus „Philosophie“ auf Denker der buddhistischen Tradition anzuwenden. Er widersetzt sich damit einem Gebrauch des Ausdrucks tetsugaku, der sich ausschließlich auf europäische und amerikanische Philosophie bezieht. Aus dem gleichen Grund nennt Nishitani seine eigene Philosophie „Standpunkt des Zen“ (j. zen no tachiba 禅の立場 ${ }^{6}$ und Ueda „Philosophie des Zen“ (j. zenteki tetsugaku 禅的哲学) ${ }^{7}$.

2 Vgl. Müller 2009: 114.

3 Nishida 1988, Vol. 11: 398-399, 420.

4 Nishida 1988, Vol. 10: 438.

5 Nishida 1988, Vol. 12: 365.

6 Nishitani 1993, Vol. 11: 3-157.

7 Ueda 2002a: 13. 
In allen genannten Fällen ist die Ansage laut und klar: Die Philosophen der Kyoto-Schule, insbesondere Nishida, Nishitani und später Ueda, bestehen nicht nur darauf, dass ihre philosophischen Systeme eine Affinität zu buddhistischem Denken aufweisen, sondern schrecken auch nicht davor zurück, „Zen“ und „Philosophie“ im selben Ausdruck zu gebrauchen. Während eine zynische Kritik dazu tendieren mag, einen solch zusammengesetzten Ausdruck so zu interpretieren, dass er nichts anderes meint als „Zen-Seele und philosophische Methode“, haben Nishitani und Ueda überzeugend dafür argumentiert, dass eine „Zen-Philosophie“ nicht nur eine Möglichkeit, sondern darüber hinaus auch eine plausible und gültige Denkoption darstellt.

Auf den folgenden Seiten werde ich erörtern, inwiefern eine solche Zen-Philosophie auch in einer akademisch akzeptablen Art und Weise gedacht werden kann, und wie Uedas dreifältiges Modell der Zen-Diskurse Kriterien und Kategorien aus der zen-buddhistischen Tradition heraus zur Anwendung bringt, um diesem Anspruch Substanz zu geben.

\subsection{Die politisch-ideologischen Implikationen}

Geht man von den geistesgeschichtlichen Tendenzen aus, wie sie zu Lebzeiten Nishidas und Nishitanis herrschten, war es vorhersehbar, dass allein schon der Gedanke an oder der Hinweis auf die Möglichkeit von Zen-Philosophie Kritik sowohl von Seiten der akademischen Philosophie als auch der Intellektuellen in Japan hervorrufen würde. Europäische und amerikanische Philosophen und Interpreten des Zen-Buddhismus haben häufig behauptet, dem Zen-Buddhismus mangele es an logischer Struktur, an ontologischen Festlegungen und einem ethischen Rahmenwerk, um sich als Philosophie zu qualifizieren.

Mit Bezug auf die Zen-Ethik wird diese kritische Haltung gerade in neuster Zeit durch Lee Stauffer besonders deutlich artikuliert und kann sehr einfach auf die Idee „Zen-Philosophie“ angewandt werden. Stauffer argumentiert: „Ethics has been variously described as: the study of good and evil, guidance about what to do and seek and how to treat other, systematic account of moral knowledge, and prescriptive discourse. All of these definitions seem to have in common the notion of one action being morally required over another [...] It is precisely this discrimination that Zen cannot make. “8 Analog zu diesem Argument wurde gezeigt, dass jede Tradition oder Disziplin, die das Prinzip der Nicht-Unterscheidung betont, unfähig ist, Wahrheit von Unwahrheit zu unterscheiden und Wahrheitswerte dem einen, aber nicht dem anderen zuzuordnen.

8 Stauffer 1989: 80. 
Auf der anderen Seite reagierten die beiden wichtigsten Popularisierer des Zen-Denkens in englischer Sprache, Suzuki Daisetsu (1870-1966) and Abe Masao (1915-2006), übereinstimmend und behaupteten, „Zen [has] nothing to teach us in the way of intellectual analysis“9, weshalb auch gelte: „[It] is not a philosophy“10. Suzuki insistiert darauf, dass „Zen“ Rationalität überschreite und ein paradoxales Paradigma notwendig mache; Abe betrachtet Zen als einen Diskurs über „absolute nothingness“ (j. zettai $m u$ 絶対無) und als die „positionless position“"11, welcher, ihm zufolge, die sogenannte westliche Tradition ermangele. In beiden Fällen wird „Zen“ als anti-intellektuell und nicht-dualistisch und folglich in einem Grundkonflikt mit jeglichem philosophischen Unternehmen betrachtet.

Letztlich jedoch ist keines der Argumente durch intellektuelle Neugier oder akademische Sorgfalt getrieben, sondern eher durch politische oder ideologische Agenden und den Versuch motiviert, eine diskursive Hegemonie zu etablieren. Die Philosophen, die meinen, dass „Zen“ nicht ausreichend Sorgfalt und Methode aufweise, die notwendig seien, um sich als Philosophie zu qualifizieren, streben danach, einen gegebenen philosophischen Kanon zu universalisieren, der - ironischerweise - geographisch und methodisch ${ }^{12}$ derart definiert ist, dass damit die Überlegenheit des westlichen Denkens geltend gemacht und die Reinheit des philosophischen Unternehmens gesichert wird. ${ }^{13}$

Andererseits kehren Suzuki und Abe den orientalisierenden Blick um und verweisen auf eine wesentliche Differenz zwischen „östlichem“ und „,westlichem“ Denken, um festzustellen, dass nur „Zen“ erschließe, was es bedeute, Mensch zu sein. In der gleichen Weise, in der einige europäische und amerikanische Denker sich daran machen zu beweisen, dass alle Traditionen Süd- und Ostasiens einer Logik oder Ethik ermangelten, interpretiert Abe die Idee des „absoluten Nichts“, welche der „Westen“ ihm zufolge so schmerzhaft

9 Suzuki 1964: 38.

10 Abe 1985: 3.

11 Abe 2003: 10.

12 In seinem Aufsatz „Tradition, Textuality, and the Trans-lation of Philosophy“ weist Maraldo darauf hin, dass Argumente ähnlich solchen, mit denen man versuchte, süd- und ostasiatische Philosophien vom philosophischen Kanon auszuschließen, dazu dienten „reine Philosophie“ vor anderen Disziplinen wie Literatur zu schützen. Ich pflichte Maraldo bei, dass auch dieses Projekt äußerst problematisch ist.

13 Um Thomas P. Kasulis' treffende Beobachtung in Hinsicht auf die akademische Suche nach einer „Zen-Ethik“ zu paraphrasieren, „the hidden assumption in the conversation is often that a tradition must, or at least should, develop some philosophical system. If Buddhism does not have a philosophical system ... it is somehow flawed ... as a tradition.“ „The second assumption is ... that a philosophical system in Buddhism ... would be immediately recognizable to a Westerner as a philosophical system“ (Kasulis 1990: 41). 
vermissen lässt, als ein Anzeichen für die intellektuelle Überlegenheit des „Buddhismus“.

Ironischerweise tappen beide Seiten in die gleiche Falle: Sie essentialisieren Zen-Buddhismus ebenso wie Philosophie und identifizieren „Zen“ mit „Un-Denken“ (c. feisiliang, j. hishiryō 非思量) und „westliche Philosophie“ mit „systematischem Denken“. Eine solche Ideologie verleugnet die Diversität und den Reichtum einer religiösen Tradition einerseits, einer akademischen Disziplin andererseits. Schließlich beanspruchen beide Seiten die Autorität festzulegen, was „reines Zen“ und was „wahre Philosophie“ darstellt.

Wenn man sich der Frage nach Zen-Philosophie annähert, ist es wichtig, nicht das höchst politische und ideologische Wesen der Fragen wie „Was ist Philosophie?“ oder „Hat Zen eine Philosophie?“ zu übersehen. Gerade die Frage, was Philosophie ist, ist letztlich eine Frage des Kanons und zielt darauf $\mathrm{ab}$, eine Axiologie $\mathrm{zu}$ etablieren und somit eine Hierarchie der Traditionen. Insofern geht es hier nicht allein um ein Problem der Methodologie, sondern der Ideologie. Das gilt insbesondere in Hinblick auf die Frage nach japanischer Philosophie: Innerhalb des Diskurses um Meiji- und Taishō-Japan diente die japanische Übersetzung von „Philosophie“, „tetsugaku“, dazu, eher eine Tradition als eine akademische Disziplin zu bezeichnen. Oder die Bezeichnungen „Zen“ und „Philosophie“ wurden beide, um die Dinge noch schlimmer zu machen, mal dazu gebraucht, eine Tradition zu bezeichnen, d.h. den ZenBuddhismus und die euro-amerikanische Philosophie, und mal dazu, eine epistemische Modalität zu bestimmen, d.h. „Un-Denken“ und „systematisches Denken“.

\subsection{Wie lässt sich die Essenzialisierung von „Zen“ und „Philosophie“ überwinden?}

Unabhängig davon jedoch, ob „Zen“ und „Philosophie“ als unwandelbare und getrennte Entitäten betrachtet, oder die Termini dazu genutzt wurden, eine Tradition bzw. eine Untersuchungsmethode zusammenzufassen, begingen die oben erwähnten Herangehensweisen an die Frage der Zen-Philosophie den Fehler, Traditionen zu essentialisieren und als unwandelbare Monolithen $\mathrm{zu}$ behandeln. ${ }^{14}$ Sie ignorierten in der Folge die Tatsache, dass sich

14 Robert Sharf argumentiert überzeugend, dass gerade die Idee des „Zen“, d.h. die Vorstellung, dass das „Zen“ ein Wesen bestimmt, „ein Konstrukt des 20. Jahrhunderts“ ist (Sharf 1994: 44-46). 
Traditionen wandeln und sich nicht klar von einander abgrenzen lassen, ${ }^{15}$ sondern vielmehr, um einen Ausdruck von Kwame Anthony Appiah zu borgen, „shape-shifting targets“"16 darstellen. Oder in den Worten Maraldos, „[s] eldom is considered the possibility that traditions are shifting entities, and that the traditionalizing of a select group of texts has become the condition for doing philosophy“17.

Wie können wir „Philosophie“ also möglichst angemessen definieren und über „Zen-Philosophie“ reden, ohne in die Fallen des hegemonialen Diskurses und des Streits um Definitionen zu tappen? Ein Aufsatz, der einen Schlüssel zu diesem Dilemma bietet, ist Maraldos „Tradition, Textuality, and the Trans-lation of Philosophy“. Um das Rätsel der japanischen Philosophie zu lösen, d.h. die Frage, ob es vor der Meiji-Zeit eine Philosophie in Japan gab, definiert Maraldo Philosophie „as an idiom of translation“18. Seine Definition basiert auf der Betrachtung, dass „texts convey philosophical methods, problems and terminology“19. Er stellt die Behauptung auf, Philosophie schließe immer die Übersetzung von Texten zwischen unterschiedlichen Perioden, Kulturen und Sprachen innerhalb einer oder zwischen verschiedenen Traditionen ein.

Maraldos ziemlich brilliante Definition hat bedeutsame Folgen für das gegenwärtige Projekt. Jede Theorie, die davon ausgeht, dass Philosophie keine ,universal tongue“ darstellt, sondern eher „on a community“ ${ }^{20}$ basiert, erkennt damit die Diversität unter und innerhalb einzelner philosophischer Traditionen und, noch wichtiger, die Existenz von ,various ,self-understandings“ [of philosophy] throughout history“'21. Das bedeutet nicht nur, dass eine recht große Chance besteht, dass Zen-Philosophie existiert, sondern auch, dass die innerhalb der zen-buddhistischen Tradition entwickelten Verständnisformen von Philosophie sich erheblich von denen anderer philosophischer Traditionen wie der analytischen Philosophie unterscheiden. Folglich kann es sein, dass Zen-Philosophie nicht „immediately recognizable to a Westerner as a philosophy“22 ist.

Der Schlüssel zur Entdeckung einer Zen-Philosophie liegt dann nicht in der Projektion notwendiger und hinreichender, in der analytischen oder kontinentalen Philosophie entwickelten Kriterien auf den Zen-Buddhismus, sondern in der

15 Für eine Diskussion der Probleme essenzialisierender Konzepte vgl. Arjun Appadurai (1996): Modernity at Large: Cultural Dimension of Globalization. Minneapolis: University of Minnesota Press.

16 Appiah 2005: 130.

17 Maraldo 1995: 226.

18 Maraldo 1995: 233.

19 Maraldo 1995: 227.

20 Maraldo 1995: 233.

21 Maraldo 1995: 233.

22 Siehe Fußnote 13. 
„Über-setzung“23 des philosophischen Idioms der zen-buddhistischen Tradition in das Idiom jeder herkömmlichen Tradition der akademischen Philosophie. Und genau dabei spielt Ueda eine zentrale Rolle. So unwahrscheinlich es erscheinen mag: Ueda bietet ein heuristisches Modell, das uns, trotz seiner Sprache, die bisweilen noch an eine orientalisierende Rhetorik erinnert, ${ }^{24}$ ein geeignetes Verständnis des Zen-Buddhismus verleiht, ohne sich einem Essentialismus oder einem hegemonialen Denken anzuverwandeln oder den Kategorienfehler zu begehen, „Zen“ und „Philosophie“ als gleichartig zu behandeln.

Es gelingt Ueda im Gegenteil, das im Zen-Diskurs entwickelte und verwendete Idiom zu „über-setzen“, und zwar in eine Sprache zu übersetzen, die insbesondere der akademischen Philosophie, aber auch dem akademischen Diskurs allgemein zugänglich ist und vice versa.

\section{Uedas Reflexion der „Zen-Philosophie“ im Horizont von Nishida und Nishitani}

Von den Philosophen der Kyoto-Schule ist Ueda der erste, der die Termini „Philosophie“ und „Zen“ konsistent und durchgängig miteinander verbindet. Um die Frage nach „Zen-Philosophie“ an sich diskutieren zu können, führt Ueda in einem ersten Schritt seine eigenen Kategorien ein und vermeidet die ziemlich aufgeladenen Termini „Philosophie“ und „Zen“. In Zen und die Welt unterscheidet er zwischen „Studium der höchsten Reflexion“ (j. kōji no hansei no gaku 高次 の反省の学) und „Praxis des Un-Denkens“ (hishiryō no gyō 非思量の行) ${ }^{25}$.

\subsection{Uedas begriffliche Mittel der Reflexion}

Seine Wortwahl ist äußerst interessant und aufschlussreich. Während das Binom „hansei“ im buddhistischen Kanon erscheint - am zahlreichsten findet es sich in Laute und Bedeutungen aller Heiligen Schriften (c. Yiqiejing yinyi; j. Issaikyō ongi 一切經音義) ${ }^{26}$-, wurde der Term am häufigsten im Japan der Meiji- und

23 Maraldo benutzt absichtlich die Bindestrich-Schreibweise „trans-lation“, um darauf hinzuweisen, dass der Akt des Philosophierens eine „Lageänderung“ innerhalb des geistigen und manchmal auch innerhalb des kulturellen Rahmens erfordert.

24 Er übernimmt eine Rhetorik, die „Ost“ und „West“ nebeneinanderstellt (Ueda 2002a: 12) und sogar vom „Geist des Ostens“ spricht (Ueda 2002a: 56).

25 Ueda 2002a: 11-12.

26 Taishō shinshū daizōkyō [im Folgenden abgekürzt als „T“] 54. 2128. 
Taishō-Zeit gebraucht, um philosophische Reflexion „westlichen Stils“ anzuzeigen. Es ist jedoch nicht „Reflexion“ im eigentlichen Sinn, die Ueda hier bestimmt, sondern vielmehr der Meta-Diskurs über die „Reflexion über die Reflexion“ ${ }^{\text {27 }}$, d.h. der Diskurs, der die Grundlage des philosophischen Denkens selbst aufklärt.

Die zweite Formulierung „hishiryō no gyō“ evoziert ganz klar die buddhistische Tradition. „Gyō“ umfasst den japanischen Ausdruck für buddhistische Praxis, während der Ausdruck „hishiryō“ seine Wurzeln im buddhistischen Kanon hat. Der letzte Begriff stellt die chinesische Übersetzung des Sanskrit-Worts acintayitvā dar, „nicht denken“ oder „nicht unterscheiden“. Während jedoch der Ausdruck j. „fushiryō“ (c. busiliang 不思量), der bisweilen ebenfalls mit „nicht unterscheiden“ oder „nicht denken“ übersetzt wird, häufig dazu dient, die Negation von j. „shiryō“ (c. siliang 思量), d.h. „denken“ oder „unterscheiden“ ${ }^{28} \mathrm{zu}$ bezeichnen - einem Term, der dazu genutzt wird, die Grundfunktion des Bewusstseins zu bestimmen - kommt der Term „hishiryō“ in den ältesten Fundstellen als alleinstehende Formulierung vor, die das Fehlen von Unterscheidungen anzeigt. ${ }^{29}$ Erst in der späteren, im Xuzangjing gesammelten Literatur, und insbesondere in Dōgens Faszikeln „Zazengi“ und „Fukan zazengi“ wird dieses „hishiryō“ im Verbund mit anderen Ableitungen von „shiryō“ gebraucht, um die berühmte Phrase zu formulieren: „Wie denkt [shiryō] man Nicht-Denken [fushiryō]? Durch Un-Denken [hishiryō].“30 In diesem Satz meint „Un-Denken“ („non-thinking“) natürlich einen Mittelbegriff zwischen Denken und Nicht-Denken („not-thinking“), ohne allerdings ihre Konnotation „nicht unterscheiden“ $\mathrm{zu}$ verlieren.

Wie dem auch sei, ich denke, es ist höchst bedeutsam, dass Ueda nicht die Begriffspaare „shiryō“ und „hishiryō“ bzw. „Reflexion“ und „Intuition“, sondern gezielt Termini auswählt, die in zwei verschiedenen intellektuellen Traditionen entwickelt wurden und für diese als fundamentale Kategorien kennzeichnend sind. Anders gesagt, er „über-setzt“ zwei Idiome, um die epistemischen Modalitäten und Untersuchungsmethoden $\mathrm{zu}$ bestimmen, die ihm als heuristisches Mittel dienen, verschiedene Arten des Zen-Diskurses zu klassifizieren. In gewissem Sinn akzeptiert Ueda durch seine terminologische Wahl, dass Philosophie

27 Ueda 2002a: 11.

28 Vgl. bspw. das Plattform-Sūtra des sechsten Patriarchen (c. Liuzu tanjing; j. Rokuso dangyō) (T 48.2007.339b) und Die Überlieferung des Lichts (c. Xu zhuandeng lu; j. Zoku dentō roku) (T 51.2077.518c).

29 Siehe das Sūtra der vollkommenen Weisheit (s. Mahāprajñāpāramitā Sūtra; c. Bore boluomi duo jing; j. Hannya haramitta kyō) (T 8.232.731a) und die Überlieferung des Lichts (T 51.2077.675b).

30 Dōgen 1969, Vol. 1: 89. 
als akademische Disziplin aus der europäischen Tradition erwachsen ist und dennoch auf andere Traditionen angewendet werden kann, ohne diesen das Wertesystem der eigenen ursprünglichen Tradition aufzuzwingen. Im gleichen Zug verschafft Ueda der epistemischen Modalität von „hishiryō“ in seiner buddhistischen Herkunft Geltung, ohne diese Kategorie auf die Tradition zu beschränken, in der sie sich entwickelt hat.

Im zweiten Schritt definiert Ueda diese Kategorien in eigenen Begriffen. Genauer gesagt zitiert er Nishidas Studie über das Gute (j. Zen no kenkyū 善の研究) ${ }^{31}$ als Inspiration und schlägt vor, dass die „Praxis des Un-Denkens“ eine „reine Erfahrung“ (j. junsui keiken 純粋経験) wiedergibt, während „das Studium der höchsten Reflexion“ den Versuch darstellt, „alles zu erklären“ (j. subete wo setsumei すべをを説明) ${ }^{32}$. Ueda übernimmt diese Formulierungen von Nishida, der in der Einleitung zu seiner Studie über das Gute erklärt, dass das Ziel bei ihrem Verfassen eben war, auf der Grundlage „reiner Erfahrung“ „alles zu erklären“. In seiner Arbeit erklärt Nishida, „dass Erfahrung bedeutet, alle Dinge so zu kennen, wie sie sind [...]. Zu sagen, dass sie rein ist, [...] fügt keinen unterscheidenden Gedanken bei, sondern weist auf die Bedingungen des Erfahrens selbst hin“33. Mit anderen Worten, „reine Erfahrung“ geht der Differenzierung in epistemisches Subjekt und Objekt vorher. Ueda beschreibt also die „Praxis des Un-Denkens“ als eine vorbewusste und vorsprachliche Erfahrung, während er den Term „Studium der höchsten Reflexion“ gebraucht, um den Versuch zu bezeichnen, systematisches Wissen über die Realität zu erlangen.

\subsection{Nishidas Idee der „reinen Erfahrung“}

Im Licht von Nishidas Definition ist es wichtig festzuhalten, dass sein Konzept von „reiner Erfahrung“ von William James’ Konzept, dem er den Begriff entlehnt, verschieden ist. Außerdem wirft die Nebeneinanderreihung von „reiner Erfahrung“ und „systematischer Erklärung“ zahlreiche methodische und philosophische Fragen auf. Während das Moment der „Nicht-Unterscheidung“ ein Thema zu sein scheint, das Nishidas „reiner Erfahrung“ und dem Gebrauch von „hishiryō“ im buddhistischen Kanon gemein ist, bleibt es natürlich äußerst problematisch, Nishidas „reine Erfahrung“ und „Zen“ generell miteinander gleichzusetzen. Erstens impliziert es eine essentialistische Konzeption von „Zen“ und „Zen-Erfahrung“, im vorliegenden Fall in der Reihung mit

31 Nishida 1988, Vol. 1: 1-199.

32 Ueda 2002a: 12.

33 Nishida 1988, Vol. 1: 9. 
„westlichem Denken“. Zweitens tappt es in die Orientalismus-Falle, die Welt in Ost und West zu teilen und „Zen“ und „westliche Philosophie“ einander entgegenzusetzen. Während Ueda gegenüber Orientalismus-Vorwürfen nicht immun ist - er identifiziert „junsui keiken“ mit „Geist des Ostens“34 und „Philosophie“ mit dem „Westen“35 - richtet sich sein Fokus mehr auf „die Praxis des UnDenkens“ und „das Studium der höchsten Reflexion“ als auf die Gegenüberstellung von „Zen“ und „Philosophie“, wodurch er die orientalisierende Rhetorik, die er manchmal gebraucht, umgeht und letztlich unterminiert.

Drittens wirft die Rede von „reiner Erfahrung“ eine Frage auf, die Takahashi Satomi in unmittelbarer Antwort auf Nishidas Studie über das Gute und Bernard Faure in neuerer Zeit noch einmal gestellt hat mit Bezug auf die Möglichkeit, eine reine Erfahrung $\mathrm{zu}$ haben und dazu noch die, auch darüber reden $\mathrm{zu}$ können. Während Faure überhaupt die Möglichkeit an sich, eine „reine Erfahrung“ zu haben, verwirft, verweist Takahashi auf die Absurdität dessen, was er als Nishidas Monismus begreift: „Wenn wir sagen, dass eine Einheit einen Gegensatz erfordert, der vereinigt ist, und ein Gegensatz eine Einheit, die den Gegensatz ermöglicht, dann stellt sogar ein Gegensatz eine reine Erfahrung dar und eine Einheit sogar eine unreine Erfahrung. “36 Hier behauptet Takahashi, dass es unmöglich sei, Kategorien zu entwickeln oder Philosophie zu betreiben, wenn man sich ernsthaft einem Monismus verschreibt.

\subsection{Uedas Rückgriff auf Nishidas Nicht-Dualismus}

Nishida jedoch versuchte sich an einem Nicht-Dualismus, der zwei Konzeptionen vermeidet: erstens einen Monismus und damit die Unfähigkeit, Unterscheidungen zu treffen; zweitens einen Dualismus, der die Welt dichotomisiert. Denn Nishida stellte die Behauptung auf, dass „Einheit“ und „Nichteinheit“ wie auch „Reinheit“ und "Nichtreinheit“ „nicht verschieden sind [...] Verschiedenheit ist immer eine Frage des Grades.“37 Später wurde ihm allerdings klar, dass sein Konzept „Selbst-Identität des absoluten Widerspruchs“ (j. zettai mujunteki jiko dōitsu 絶対矛盾的自己同一) besser als die Idee der „reinen Erfahrung“ dazu geeignet war, einen Nicht-Dualismus $\mathrm{zu}$ formulieren, der weder epistemische Modalitäten noch kulturelle Haltungen essentialisiert. Dieser terminologische

34 Ueda 2002a: 56.

35 Ueda 2002a: 39. Er nimmt an, dass sich „westliche Philosophie“ „in Richtung auf das Nichts“ entwickelt, während die „östlichen Traditionen“ „aus dem Nichts“ hervorgehen.

36 Takahashi 1973, Vol. 4: 169.

37 Nishida 1988, Vol. 1: 16; Nishida 1988, Vol. 15: 91. 
Wechsel ebnete den Weg für Nishidas späte Philosophie, welche die Dichotomie von Erfahrung und Reflexion und, wenn konsistent angewendet, die von „Ost“ und „West“ untergräbt.

Meines Erachtens übernimmt Uedas Modell diese nicht-dualistische Lesart der Idee der „reinen Erfahrung“ und damit folglich das subversive Potential Nishidas späterer Terminologie. Ich werde das Moment der Subversion, das Nishidas Nicht-Dualismus eigen ist, weiter unten diskutieren. Um für diese These zu argumentieren, möchte ich jedoch zu Uedas Modell zurückkehren.

Während Ueda Nishidas Terminologie der „reinen Erfahrung“ als die begriffliche Basis seiner eigenen philosophischen Untersuchung bestimmt und an anderer Stelle ${ }^{38}$ auch dafür argumentiert, dass Nishidas Tagebücher und Briefwechsel nahelegen, dass Studie über das Gute eine Zen-Philosophie enwirft, ist sich Ueda durchaus bewusst, dass es aller Affinität zwischen Zen-Ideen und Nishidas späterem Werk zum Trotz problematisch ist, Nishida selbst einen Zen-Philosophen zu nennen. Ueda selbst bestimmt Nishidas Philosophie eher als eine „Philosophie der Philosophie“ (j. tetsugaku no tetsugaku 哲学の哲学) ${ }^{39}$ denn als eine „Philosophie des Zen“40. Es ist problematisch, sich auf Nishidas Denken in Studie über das Gute als eine Zen-Philosophie zu beziehen, weil Nishida die Beziehung zwischen Zen-Ideen und seiner Terminologie der „reinen Erfahrung“ an keiner Stelle in Studie über das Gute weder begründet noch evoziert. ${ }^{41}$

Erst wesentlich später (1938-1945) diskutiert Nishida nicht nur buddhistische Ideen in seinen Briefwechseln und Tagebüchern, sondern führt auch Zitate buddhistischer Texte in seinen philosophischen Schriften an. Während Nishida die meiste Zeit seiner Laufbahn dazu neigte, alle Philosophien in die drei Kategorien Objektivismus, Subjektivismus und Nicht-Dualismus zu klassifizieren, ${ }^{42}$ kommt es erst nach 1938 dazu, dass er die dritte Ebene, die er zuvor mit Hegels Dialektik oder Boehmes Mystik illustrierte, ${ }^{43}$ mit dem Buddhismus identifiziert. ${ }^{44}$ Für Nishida ist buddhistische Philosophie im Allgemeinen

38 Siehe zum Beispiel sein „Nishida kitarō wa dare ka“, Ueda 2002b.

39 Ueda 2002a: 57.

40 Ueda 2002a: 13.

41 Ueda selbst bemerkt, dass „es möglich ist zu sagen, dass das Denken in Studie über das Gute tief beeinflusst ist durch Zen; nichtsdestotrotz müssen wir betonen, dass Zen darin nicht auftaucht“" (Ueda 2002a: 55).

42 Ich argumentiere für diese Behauptung ausführlicher in meinem Aufsatz „The SelfContradictory What?-Reflections on how to Teach the Philosophy of Nishida Kitarō" (Kopf 2008).

43 Siehe Nishida 1988, Vol. 2: 181-182.

44 Siehe Nishida 1998, Vol. 11: 371-468. Ueda macht auch die Beobachtung, dass Nishida Zen an seine Vorstellung von „Selbst-Identität des absoluten Widerspruchs“ und „inverse 
und Zen-buddhistisches Denken im Besonderen paradigmatisch für den NichtDualismus, auf den er selbst hinarbeitete und dem er schließlich 1937 in seinem Aufsatz „Kōiteki Chokkan“45 die begriffliche Form geben konnte. Genau aus diesem Grund ist Uedas Beschreibung Nishidas als „Philosoph, der auch ein Praktiker des Zen war“46 angemessener als die Bezeichnung „Zen-Philosoph“.

Während Nishidas Werk mit der „Grundlegung von Philosophie“ beginnt und mit dem „Ende der Philosophie“ schließt, beginnt und endet Nishitanis Werk mit Zen. ${ }^{47}$ Aus diesem Grund führt Ueda, so sehr er sich auch auf Nishidas Studie über das Gute als Quelle und Inspiration stützte, die Philosophie Nishitanis als Modell für eine Zen-Philosophie an. Ueda erklärt, dass „Nishitani mehr als Nishida, wie sein Vertrauen auf die Leere als seine Grundkategorie veranschaulicht, vom [Standpunkt der] Tradition des Mahāyāna-Buddhismus aus denkt"“48. Und in der Tat, Nishitani hat nicht nur einen Kommentar zu Dōgens Shōbōgenzō, ${ }^{49}$ zum HerzSūtra und zu grundlegenden buddhistischen Konzeptionen geschrieben, sondern auch seinen eigenen philosophischen Standpunkt entwickelt, den er, ausgehend von den sogenannten „vier Prinzipien des Zen“, als „Standpunkt des Zen“ bezeichnet.

\subsection{Anschlussmöglichkeiten an Nishitani}

Das Kennzeichen des „Standpunkts des Zen“ ist für Nishitani, ${ }^{50}$ dass es „die Untersuchung in die Sachen des Selbst“ (j. koji kyūmei 己事究明) zu seinem Hauptanliegen macht - er übernimmt diesen Ausdruck von dem als Daitō Kokushi (1282-1337) ${ }^{51}$ bekannten japanischen Zen-Meister. Nishitani entwickelt diese Philosophie der Selbst-Erkenntnis durch die Verwendung der Terminologie

Korrelation“ angleicht (Ueda 2002a: 59). Beide Konzepte entwickelte Nishida in den letzten sieben Jahren seines Lebens.

45 Nishida 1988, Vol. 8: 541-571.

46 Ueda 2002a: 16.

47 Nishitanis eigene Terminologie verwendend erklärt Ueda, „Nishitani [...] nennt Zen ,das Vorher und Nachher der Philosophie““, während „das ,Vorher‘ im Fall von Nishida in der Fundierung der Philosophie und das Nachher im ,Ende der Philosophie‘ liegt“ (Ueda 2002a, Vol. 5: 37).

48 Ueda 1997: 45.

49 Vgl. Müller 2016.

50 An anderer Stelle folge ich Nishitanis Vorschlag, das Prinzip der Selbst-Reflexion als einen möglichen Weg zur Definition von Philosophie zu nutzen (Kopf 2015a: 142-144).

51 Der Ausdruck „die Sache des Selbst“ (c. jishi; j. koji 己事) genauso wie die Phrase, „die Sache des Selbst untersuchen“ (c. jiuming jishi; j. kyūmei koji究明已事) kommen beide mit einer gewissen Häufigkeit in der Zen-Literatur vor. 
und Einsichten Descartes’ und des Yogācāra Buddhismus. Kurz, Nishitani interpretiert die Ideen, die gewöhnlich als die „vier Prinzipien des Zen“ bezeichnet werden - „es gibt eine Überlieferung außerhalb der Schriften“, „mach Dich nicht abhängig von Worten“, „zeige unmittelbar auf das Herz der Person“, „werde Buddha, indem Du Deine eigene Natur siehst“ - so, dass sie zur Selbst-Reflexion und Selbst-Analyse antreiben; und zwar ganz im Sinn von Descartes' wie auch Sokrates' dictum „Erkenne Dich selbst“.

Zumeist wurden die vier Prinzipien, ${ }^{52}$ die im Zen-Kanon äußerst verbreitet $\operatorname{sind}^{53}$ und dem legendären Schulgründer Bodhidharma zugeschrieben werden, als Postulat interpretiert, dass die Erfahrung des „die eigene Natur Sehens“ (c. jianxing; j. kenshō 見性) und die Überlieferung „von Angesicht zu Angesicht“ (c. mianshou; j. menju 面授) der Reflexion und Sprache überlegen sind. Nishitani jedoch interpretiert diese vier Redeweisen so, dass sie die Postulate von Introspektion und Selbst-Erkenntnis formulieren. Wie Descartes, so argumentiert Nishitani, verweisen diese Zeilen auf eine tiefere, psychologische Ebene jenseits des selbst-bewussten Ichs. Anders als Descartes jedoch glaubt Nishitani, dass diese Introspektion die Ebene des Bewusstseins eröffnet, die im Yogācāra Buddhismus als das „Speicherbewusstsein“ (s. ālayavijñāna; c. aliyeshi; j. ariyashiki 阿賴耶識) bezeichnet wird und die fundamentale Leere aller Dinge, das Selbst eingeschlossen, offenbart.

Für die gegenwärtige Diskussion ist jedoch nicht seine Philosophie der Leere wichtig, es ist vielmehr beachtenswert, dass Nishitani nicht nur erfolgreich Denker, Texte und Konzepte aus der buddhistischen Tradition in den Diskurs der akademischen Philosophie einführt, sondern auch einen neuen philosophischen Standpunkt entwickelt in der „Über-setzung“ zweier Idiome, und zwar das der „der Untersuchung der Sachen des Selbst“ und das der vier Prinzipien, welche beide für die buddhistische Tradition zentral sind. Es ist wichtig, sich bewusst $\mathrm{zu}$ machen, worin genau Nishitanis Projekt bestand, wenn wir Uedas Vision einer Zen-Philosophie und sein Modell dreier grundlegender Diskurse unter diesen Denkern der Kyoto-Schule untersuchen, die an systematischer Reflexion auf die Zen-buddhistische Theorie und Praxis interessiert waren.

52 Die sogenannten „vier Prinzipien des Zen“ werden gewöhnlich Bodhidharma zugeschrieben, werden aber wahrscheinlich sehr viel später entwickelt. Für eine historische Diskussion vgl. Morten Schluetters How Zen Became Zen (Schluetter 2010).

53 Siehe als Beispiel die Aufzeichnugen des Zen-Meisters Linji (c.: Linjilu; j. Rinzairoku) (T 47.1985.495b). 


\section{Drei Formen des Zen-Diskurses zwischen Praxis und Philosophie ${ }^{54}$}

In Zen und die Welt ${ }^{55}$ unterscheidet Ueda drei Arten des Diskurses innerhalb der Zen-Tradition: „Zen“ (j. zen 禅), „Zen-Denken“ (j. zenshisō 禅思想) und „Philosophie“ (j. tetsugaku 哲学). Die erste Art des Diskurses betrifft die „Praxis des Zen“ (j. zenshugyō 禅修行) und die „konkrete Erfahrung“ (j. gutaiteki na keiken 具代的 な経験), die zweite das „Verständnis des Zen“ (j. zenrikai 禅理解) und die dritte das „Verständnis der Welt“ (j. sekairikai 世界理解)

\subsection{Drei Diskurse und drei Denker}

Eine „Zen-Person“ (j. zensha 禅者) ist allein an der Praxis interessiert, die zum Selbst-Erwachen und zu „reiner Erfahrung“ führt ${ }^{57}$, welche Ueda beide im Herzen der Zen-Praxis verortet. Die Aufgabe einer Zen-Person ist es, eine Analyse der Erfahrung des Erwachens zu verbalisieren und darzubieten. Ueda bestimmt als den Hauptrepräsentanten eines solchen Projekts Hisamatsu Shin'ichi (18891980), ein Zen-Meister und Schüler Nishidas.

Ein Zen-Denker (j. zenshisōka 禅思想家) ist jemand, der die „reine Erfahrung als die einzige Realität“ interpretiert ${ }^{58}$ und das Selbstgewahren reflektiert. Als solches ist er/sie an einer Art Reflexion zweiter Ordnung und an konzeptuellen Strukturen interessiert. Was „Zen-Denken“ vom „Zen“ unterscheidet, ist dass Ersteres den Prozess der Theoretisierung (j. shisōka 思想化) oberhalb und jenseits der Verbalisierung von „reinen Erfahrungen“ fordert, die für „Zen“ chararakteristisch sind. Der Unterschied zwischen „Zen-Denken“ und „Zen“ scheint unerheblich zu sein, aber ich werde noch auf diesen Unterschied zurückkommen. Ueda bezeichnet D.T. Suzuki, Nishidas lebenslangen Freund und der wichtigste Popularisierer des Zen-Buddhismus in der englischen Sprachwelt, als den Prototypen des Zen-Denkers.

54 Dies ist die Erweiterung eines Abschnitts aus „Towards a Conception of Philosophy as Expression: Approaching Intercultural Philosophy from a Zen Buddhist paradigm“ (Kopf 2015b). 55 Für ein anderes Herangehen an den gleichen Text vgl. Steffen Dölls „Ueda Shizuteru’s Phenomenology of Self and World: Critical Dialogues with Descartes, Heidegger, and Merleau-Ponty“ (Döll 2011).

56 Ueda 2002a: 6-7.

57 Ueda 2002a: 68.

58 Ueda 2002a: 69. 
Schließlich ist ein „Philosoph“ (j. tetsugakusha 哲学者) innerhalb der ZenTradition jemand, der wie Nishitani - ein Student Nishidas und zweiter Nachfolger der Kyoto-Schule - eine Philosophie des Selbstgewahrens entwickelt. Das Ziel einer Zen-Philosophie ist es, „alles zu erklären“ (j. subete o setsumei む゙てを 説明 $)^{59}$, d.h. die Zen-Erfahrung systematisch zu reflektieren, und zwar durch die Aneignung der Methodologie, Terminologie und Agenda, die für die philosophische Tradition, wie sie sich in Europa entwickelt hat, charakteristisch ist.

Diese drei Standpunkte stellen nicht drei separate Vorhaben dar, sondern skizzieren vielmehr einen Prozess der Objektifizierung von „Zen“ in Richtung „Philosophie“ und einen Prozess der Konkretisierung in umgekehrter Richtung. Die Zen-Person bewegt sich von der „reinen Erfahrung“ zum systematischen Denken und der „Philosoph“ vom Denken zur Erfahrung. Zugleich bedeutet das Fortschreiten vom „Zen“ zur „Philosophie“ eine fortschreitende Öffnung zur Welt und eine Vertiefung des Selbstgewahrens (j. jikaku 自覚). Bei alldem ist es wichtig $\mathrm{zu}$ beachten, dass es Ueda nicht darum geht, drei Disziplinen, sondern vielmehr drei flüchtige Eindrücke oder Zwischenschritte eines bilateralen Prozesses zu unterscheiden: den einer fortschreitenden „Objektivation“ (j. kyakkanka 客観化) vom „Zen“ zur „Zen-Philosophie“ und den einer fortschreitenden „Subjektivation“ (j. shukanka 主観化) in umgekehrter Richtung ${ }^{60}$. Außerdem behauptet Ueda, dass der erstere Prozess durch „eine Zen-Person, die Philosophie betreibt“61 und der letztere durch „einen Philosophen, der

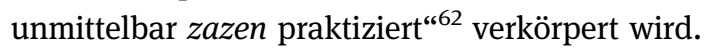

Diese Spezifikation erweitert Uedas Typologie um eine zusätzliche Dimension: Die drei Standpunkte skizzieren nicht nur drei Formen des Zen-Diskurses, sondern auch drei Aktivitäten, „Philosophie zu betreiben“ wie auch drei Modalitäten des Selbstgewahrens. „Zen“, das Ueda auch als Erwachen (j. kaku 覚) paraphrasiert, bietet eine „Grundlegung“ (j. kongen 根源) des Selbstgewahrens, „Zen-Denken“ erhellt das Selbstgewahren im eigentlichen Sinn und „Philosophie“ spiegelt das Verständnis des Selbst (j. jikorikai 自己理解) wie auch der Welt und letztlich auch das „Verständnis des Verständnisses“ (j. rikai no rikai 理 解の理解) wider. In den folgenden Abschnitten möchte ich diese drei Modalitäten „Philosophie zu betreiben“ und „seiner selbst gewahr zu werden“ etwas ausführlicher untersuchen. Dabei sollte jedoch beachtet werden, dass ich Uedas Lektüre von Hisamatsu, Suzuki und Nishitani präsentiere - nicht meine eigene.

59 Ueda 2002a: 70.

60 Ueda 2002a: 18.

61 Ueda 2002a: 72.

62 Ueda 2002a: 76. 


\subsection{Uedas Lektüre von Hisamatsu}

„Zen-Personen“ wie Hisamatsu, argumentiert Ueda, machen das „Existentielle“ (j. jitsuzonteki 実存的) und das „Praktische“ (j. jissenteki 実践的) zu ihrem fundamentalen Anliegen. Wie oben angegeben, fokussiert eine „Zen-Person“ die „Praxis des Zen“ und die „konkrete Erfahrung“. Diese Definition ist auch ein Echo auf Nishidas Definition der „reinen Erfahrung“ als das Kennen der „Dinge, wie sie sind“ - während „Erfahrung“ einen Ausdruck darstellt, der dem Idiom der Zen-Tradition fremd ist, ist der Rekurs auf den Ausdruck „Dinge, wie sie sind“ als solches der Zen-Rhetorik und -Literatur nicht unbekannt. In der Zen- Tradition wurde der Ausdruck „Dinge, wie sie sind“ oftmals so interpretiert, dass er das ineffabile bezeichnet, oder das, was, um das zweite der vier Prinzipien des Zen zu paraphrasieren, nicht auf Worten beruht. ${ }^{63}$

Ein Blick auf Hisamatsus Rhetorik verifiziert Uedas Beschreibung. Hisamatsu definiert die „besondere Methode“ des Zen als die Einsicht, dass „wir selbst aufhören zu existieren“. Oder konkreter, „ob wir es als ,kungfu“ [c. gongfu; j. kufū 工夫, Anm. d. Verf.], oder zazen bezeichnen und logische Spekulationen und moralisches Handeln zurückweisen, dies ist die Methode, welche die reale Selbstheit des Selbst, das spekuliert und handelt, in ,Nichts“ verwandelt“64. Das Konzept, auf das sich Hisamatsu als „östliches Nichts“ (j. tōyōteki mu 東洋的無) bezieht, stellt „den besonderen Charakter“ dar, „den die östliche Kultur von der westlichen unterscheidet“65. Überraschender ist jedoch, dass Ueda sich nicht auf diesen Aspekt in seiner Beschreibung konzentriert, sondern vielmehr dazu übergeht, eine diskursive Analyse zu Hisamatsus Werk zu geben.

Was aus Hisamatsu den Prototyp einer Zen-Person macht, so Ueda, ist nicht eine spezifische Interpretation der Zen-Texte, sondern eher, dass er in das Idiom des Zen selbst eingeschlossen ist und andere Diskurse völlig zurückweist. Während es so zu sein scheint, dass der späte Hisamatsu sich für andere Idiome öffnet, indem er Formulierungen benutzt wie „der Weg des absoluten Subjekts“, „aktives Subjekt“ und das „formlose Selbst“, ist sein Gebiet eindeutig der ZenDiskurs, und sein Denken könnte durch das charakterisiert werden, was Ueda einen „Monismus des Zen“66 nennt. Der Term „Monismus“ darf hier nicht so

63 Die sogenannte „Blumen-Predigt“, d.h. der sechste Fall der Torlosen Schranke (T 48.2005) das die beiden ersten der vier Prinzipien des Zen enthält - wurde manchmal, wie im Fall von Shibayama Zenkei so verstanden, dass es die Doktrin formuliert, dass die Erfahrung des Erwachens, die für den Zen-Buddhismus zentral ist, ,jenseits der Worte“ liegt.

64 Hisamatsu 1969: 23.

65 Hisamatsu 1969: 29.

66 Ueda 2002a: 25. 
verstanden werden, dass er ein metaphysisches System anzeigt, sondern vielmehr, dass er einen methodischen Solipsismus oder Exklusivismus bezeichnet, der Ueda zufolge das Denken und die Methoden von Denkern und Texten meidet, die der Zen-Tradition äußerlich sind.

Hisamatsu weist also nicht per se sprachliche Ausdrücke zurück, sondern vor allem Ideen und Eigenschaften der „westlichen Kultur“ als unfähig, der „reinen Erfahrung“ Gerechtigkeit widerfahren zu lassen, und greift nach der Sprache der „grundlegenden kōan“ (j. kihōnteki kōan 基本的公案) und auch der Rhetorik der Negation, wie sie durchgehend in den Texten der Zen-Tradition gebraucht werden. Von zentralem Interesse für die gegenwärtige Diskussion ist, dass das Kriterium des „Zen“ primär die Form des Diskurses betrifft und nicht die Ideologie. „Zen-Personen“, so Uedas Analyse, beschränken sich selbst auf Idiome, die in der Zen-Tradition entwickelt wurden, weigern sich, diese zu „über-setzen“ und betonen so die Dichotomie zwischen „Ost“ und „West“. ${ }^{67}$ Diese Haltung gerät allerdings in Konflikt mit dem Nicht-Dualismus, den eine „Zen-Person“ wie Hisamatsu zu propagieren scheint. ${ }^{68}$

\subsection{Uedas Lektüre von Suzuki}

Suzuki versucht seinerseits die Dichotomisierung zwischen Erfahrung zum einen und Sprache wie auch philosophischem Diskurs zum anderen zu überwinden. Ueda legt nahe, dass Suzukis Denken die fundamentale Annahme zugrunde liegt, dass Erfahrung und Theorie nicht notwendigerweise miteinander konfligieren müssen, sondern in die gleiche Richtung auf das Selbstgewahren weisen. Oder wie es Suzuki selbst formuliert: „Zen-Denken ist bereits Zen-Handeln und Zen-Handeln ist bereits Zen-Denken." ${ }^{\text {"69 }}$ Ueda zufolge war Suzuki der Überzeugung, dass „Zen“ im engeren Sinn von Hisamatsus Unternehmen Worte erfordert und also die Erschaffung eines „Zen-Denkens“ notwendig macht: Ueda erklärt, dass „die Tatsache, dass Zen durch Worte verbalisiert werden kann [...] und die Tatsache, dass das, was durch Worte ausgedrückt wird, für den Standpunkt des Zen nicht bedeutungslos ist [...] [Diese Tatsachen] konstituieren die Grundlage des Zen-Denkens.“70

67 Hisamatsus orientalisierende Rhetorik, veranschaulicht durch den Ausdruck „östliches Nichts“, findet sich fast im gesamten Artikel, der unter diesem Titel erschienen ist, vgl. Hisamatsu 1969, Vol. 1: 11-81.

68 Hisamatsu bezieht sich auf das „östliche Nichts“ als j. ittaifuni 一体不二 „eine Realität, nicht zwei“ (Hisamatsu 1969, Vol. 1: 41).

69 Suzuki 2000: 11.

70 Ueda 2002a: 30. 
Ueda argumentiert, dass Suzuki sein eigenes Kategoriensystem entwickelt, um die Notwendigkeit eines „Zen-Denkens“ herzustellen. Daher unterscheidet Suzuki zwischen „Zen-Erfahrung“ (j. zen taiken 禅体験), „systematischem Denken“ und „Zen-Bewusstsein“ (j. zen ishiki 禅意識). Das Erstere betont die „Nicht-Unterscheidung“ (j. mufunbetsu 無分別), das Zweite die „Unterscheidung“ (j. funbetsu 分別), und das Letzere die „Unterscheidung der NichtUnterscheidung“ (j. mufunbestu no funbetsu 無分別の分別). „Zen-Erfahrung“ spiegelt „Weisheit“ (j. chi 智), systematisches Denken „Erkenntnis“ (j. chi 知), und „Zen-Bewusstsein“ „Selbstgewahren“. Bereits die Beschreibung dieser drei epistemischen Modalitäten impliziert eine klare Hierarchie.

Ueda erklärt, dass, „während es Suzukis Idiosynkrasie ist, das Herz der ZenErfahrung zu seiner Prämisse zu machen, ,wenn Erfahrung nicht bewusst wird, das heißt, wenn sie nicht durch Worte bewusst gemacht wird, dann existiert sie nicht““71. „Zen-Bewusstsein“ führt zur höchsten Form des Wissens, d.h. zum Selbstgewahren, und es „schließt sogar den Standpunkt der Reflexion ein““72.

Der Hauptunterschied zwischen Suzuki und Hisamatsu ist jedoch nicht ein ideologischer - beide betonen, jeder auf seine Weise, die Zentralität eines nichtdualen Paradigmas für ihr System ${ }^{73}$ - sondern, so argumentiert Ueda, ein Unterschied des Idioms. Während Hisamatsu alle Diskurse, die der Zen-Tradition äußerlich sind, zurückweist, wendet sich Suzuki dem „Westen“ und der „Philosophie“ zu. Ueda betont die Tatsache, dass Suzuki in den USA gelebt und mit einer Amerikanerin verheiratet war, um seine Behauptung zu veranschaulichen, dass Suzuki „in der Welt lebte“: „Suzuki selbst, der die Welt verlassen hatte und in der Welt lebte, war dessen gewahr, dass er die Bedeutung des Zen durch die Art und Weise, wie er lebte, veränderte.“74

An dieser Stelle ist es wichtig, die Gegenüberstellung von „Welt“ und „Zen“ zu kommentieren, die in Uedas Argument implizit ist. Im Buddhismus wird das „Nonne oder Mönch werden“, „Eintritt in ein Kloster“ oder „zazen betreiben“ gewöhnlich als ein „Verlassen der Welt“ oder, wörtlich, „Verlassen des Heims“ (出家 shukke) paraphrasiert. Ueda selbst verbindet mit „Zen“ die „Praxis des Un-Denkens“ und mit der Welt „das Studium der höchsten Reflexion“. Um sein Argument in Zen und die Welt zu entfalten, übernimmt er außerdem diese Bestimmung der Welt als den „Westen“, im Fall von Hisamatsu, und als die

71 Ueda 2002a: 29.

72 Ueda 2002a: 29.

73 Suzuki nennt dieses Paradigma „Logik des ist-nicht“ (j. sokuhi 即非) und gibt ihm die logische Form von „A ist nicht-A“ (Suzuki 1968: 381), während Hisamatsu Ausdrücke gebraucht, in denen er Gegensätze mit dem Wort „ist“ (j. soku 即) verbindet (Hisamatsu 1969, Vol. 1: 30). 74 Ueda 2002a: 32. 
„Welt des Englischen“"75, im Fall von Suzuki, um es schließlich zusammenfallen $\mathrm{zu}$ lassen. Wie ich weiter unten diskutieren werde, entstehen daraus interessante Implikationen in Hinsicht auf die Gegenüberstellung der „Praxis des Un-Denkens“ mit der Welt des „Studiums der höchsten Reflexion“.

Im Moment ist es wichtig Uedas Annahme zu beachten, dass Suzuki versucht, die Lücke zwischen „Zen“ und der „Welt“ insofern zu überbrücken, als seine Vorstellung von der „Unterscheidung der Nicht-Unterscheidung“ „eine Forderung nach Ost und West einschließt“76. In diesem Prozess versucht Suzuki nicht nur zwei Traditionen, sondern auch die beiden Paradigmen der „reinen Erfahrung“ und der reinen Reflexion miteinander in Einklang zu bringen. Die Folgen seiner methodologischen Strategie sind begrifflich weitreichend. Wenn Erfahrung und Reflexion als diametral entgegengesetzt betrachtet werden, dann bringt eine Reflexion auf das ineffabile notwendig ein Paradox hervor, d.h. es erfordert, was Suzuki die „Unterscheidung der Nicht-Unterscheidung“ und seine berühmte „Logik des ist-ist nicht" ${ }^{\text {“77 }}$ nennt. Suzuki selbst lässt keine Zweifel an seiner Sichtweise: „Zen Denken wird durch Schlagworte und Phrasen ausgedrückt, wie die des Wissens des Nicht-Wissens, des Denkens des Un-Denkens, des Geistes des Nicht-Geists, des Bewusstseins des Nicht-Bewusstseins, der Unterscheidung der Nicht-Unterscheidung, der Korrelativität des Unbezüglichen, der ungehinderten Durchdringung der Erscheinungswelt und der Ähnlichkeit der zehntausend Dharmas.“78

Während Formulierungen wie diese oft als eine Zurückweisung von Rationalität und Logik interpretiert werden, argumentiert Ueda, dass sie kein Hinweis auf eine inhärente Irrationalität oder Arationalität des „Zen-Denkens“ darstellen denn am Ende kann alles „Zen-Denken“ entwickelt werden - sie sind eher eine Spiegelung dessen und eine Notwendigkeit davon, dass Suzuki versucht, zwei Paradigmen in Einklang zu bringen, ,reine Erfahrung“ und systematisches Denken, welche als polare Gegensätze konstruiert wurden, und zwischen zwei Sphären zu vermitteln, „Zen“ und der „Welt“, die als sich wechselseitig ausschließend definiert wurden. In gleicher Weise könnte man argumentieren, dass Suzukis Standpunkt mitten zwischen zwei Diskursen und sein Versuch, zugleich innen und außen zu sein, es für ihn notwendig machen, paradoxale Sprache einzusetzen. Dieser Punkt ist genauer zu durchdenken.

Natürlich entsteht die Frage, ob „Zen-Denken“, d.h. Theorie über Erfahrung, inhärent paradoxal ist oder ob das Paradox einfach ein Hinweis auf den Fehler ist,

75 Ueda 2002a: 33.

76 Ueda 2002a: 32.

77 Suzuki 1968: 381.

78 Suzuki 2000: 10-11. 
„Erfahrung“ und Theorie oder Sprache als sich wechselseitig ausschließende Termini zu definieren. Ich werde weiter unten auf diese Frage eingehen. Im Moment reicht es zu beachten, dass Ueda nahezulegen scheint, dass in der gleichen Weise, in der Hisamatsus Rhetorik der Negation aus der Zurückweisung von Paradigmen hervorgeht, die mit dem Zen-Idiom inkompatibel sind, Suzukis Rhetorik des Paradoxes aus dem Versuch erwächst, über diskursive Grenzen hinweg zu „über-setzen“ und das Zen-Idiom als ein neues Paradigma anzunehmen.

\subsection{Uedas Lektüre von Nishitani}

„Philosophen“ wie Nishitani sind letztlich darauf festgelegt, so Ueda, ,alles zu erklären“, d.h. systematisches Denken zu betreiben. Systematisches Denken ist jedoch nicht in sich selbst genügsam. Ganz im Gegenteil, Ueda legt nahe, dass Nishitani die wechselseitige Abhängigkeit von „Zen-Handeln“ und „Zen-Denken“ bis zu ihrer logischen Konsequenz treibt: „Wenn man, wie Nishitani, in der gegenwärtigen Welt lebt und gleichzeitig in der Tradition des Ostens steht, ist es essentiell, dass Zen danach strebt, ,Zen und Philosophie' miteinander zu verbinden; anders formuliert, wenn Zen westliche Philosophie akzeptiert, dann wird Philosophie von einem Standpunkt, der auf der Verbindung zwischen ,Zen und Philosophie“ errichtet wird, betrieben und zugleich revidiert." ${ }^{79}$ Und noch konkreter, „Zen, für Nishitani, konstituiert ,seinen eigenen Weg zum philosophischen Selbstgewahren““80 . Kurzum, „Philosophie“ braucht „Zen“ und vice versa.

Das ist jedoch noch nicht die ganze Geschichte. Wie schon im Fall von Hisamatsus „Zen“ und Suzukis „Zen-Denken“ betont Ueda, dass die Eigenschaft Nishitanis Projekts nicht ein spezifisches Verständnis des Zen oder eine idiosynkratische Ideologie ist, sondern vielmehr seine Verortung innerhalb der diskursiven Landschaft. Was Nishtani gegenüber Suzuki wie auch Nishida als einen „Zen-Philosophen“ qualifiziert, ist die Tatsache, dass er Philosophie vom Standpunkt des „Zen“ aus betreibt. Ueda skizziert Nishitanis Projekt wie folgt: „Es ist nicht so, dass die Frage vom Osten her gestellt wird, vielmehr beginnt man mit einem allgemeinen Problem und womöglich macht man die Antwort aus dem Osten zu einer gemeinsamen Antwort." 81 Nishitanis Diagnose ist, dass die gegenwärtige Welt an Nihilismus leidet und dass dieser Nihilismus das

79 Ueda 2002a: 38.

80 Ueda 2002a: 38.

81 Ueda 2002a: 48. 
Fundament der gegenwärtigen Zivilisation darstellt. ${ }^{82}$ Er legt nahe, dass Nihilismus mithilfe des Selbstgewahrens, das auf einer Einsicht in die Leere (j. ku 空) basiert, geheilt werden kann.

\subsection{Uedas Vision einer Weltphilosophie}

Ueda betont, dass Nihilismus für Nishitani ein „allgemeines“ Problem ist und in einem globalen Kontext angegangen werden muss. Ueda behauptet, dass „Nihilismus nicht nur das Ende der Geschichte der westlichen Philosophie darstellt, sondern auch die Bedingung der Geschichte der Weltphilosophie hervorbringt“83. Der Rahmen von Nishitanis Projekt ist die „eine Welt“ (j. hitotsu no sekai 一つの世界) - wie Nishitani beobachtet, „ist die Bedingung der jetzigen Zeitphase, dass die östlichen und die westlichen Welten sich rasend schnell zu einer Welt konsolidieren“84 - sein Ziel ist eine „Weltphilosophie“ (j. sekai tetsugaku 世界哲学) ${ }^{85}$. Der Schlüssel zu einer „Zen-Philosophie“ und einem „philosophischen Zen“ (j. tetsugakuteki zen 哲学的禅) ${ }^{86}$, nimmt Ueda an, liegt in der Vision einer „Weltphilosophie“, die das Selbstgewahren der „einen Welt“ artikuliert.

Wenn wir die offensichtlichen Probleme dieser Argumentation beiseite lassen, d.h. die Gleichsetzung von „Osten“ und „Zen“ bzw. von „Westen“ und „Philosophie“ auf der einen Seite, und die Implikation, dass allein die Verbindung von „Zen“ und „westlicher Philosophie“ ohne den Beitrag der, unter anderem, islamischen oder konfuzianischen Philosophie eine „Weltphilosophie“ erschafft, stellt Ueda drei faszinierende und wichtige Punkte heraus: 1) Die Lösung zu globalen Problemen erfordert eine globale Lösung, eine „Weltphilosophie“; 2) Diese „Weltphilosophie“ stellt eine neue Tradition dar, eine dritter Term sozusagen, und stützt sich auf multiple Traditionen; 3) Sie überwindet die Dichotomisierung der Welt in „Ost“ und „West“ (wie auch „Nord“ und „Süd“) und macht, im Fall eines Dialogs zwischen Denkern der Traditionen des ZenBuddhismus und der akademischen Philosophie, eine „Zen-Philosophie“ oder wenigstens ein „philosophisches Zen“ notwendig.

In gewissem Sinn scheint Ueda vorzuschlagen, dass die Begegnung von ZenBuddhismus und euro-amerikanischer Philosophie - um einen Ausdruck von

82 Nishitani 1993, Vol. 11: 161.

83 Nishitani 1993, Vol. 11: 161.

84 Nishitani 1993, Vol. 11: 6.

85 Ueda 2002a: 49.

86 Ueda 2002a: 13. 
Rita Gross zu borgen - eine „vielversprechende Koinzidenz“87 darstellt, weil sie die Möglichkeit einer „Weltphilosophie“ eröffnet. Diese gewagte Feststellung impliziert weiterhin, dass die „Praxis des Un-Denkens“ und das „Studium der höchsten Reflexion“ nicht notwendigerweise zwei sich wechselseitig ausschließende epistemische Modalitäten darstellen. Was Nishitani in den Augen Uedas von Suzuki unterscheidet, ist dass Letzterer sich zwei Welten vorstellt, die vermittelt werden müssen und dessen Idiome über-setzt werden müssen, um Kommunikation $\mathrm{zu}$ ermöglichen, während Ersterer seine Analyse auf dem Begriff der „Leere“ basiert und daher, zumindest in der Theorie, fähig ist, über die „vorläufigen“ Unterscheidungen hinauszugehen, die Dichotomisierung von „Ost“ und „West“ zu unterlaufen und sich „eine Welt“ vorzustellen. „Übersetzung“ schließt also nicht bloß die wörtliche Übersetzung von Konzepten von einem Idiom in ein anderes ein, sondern eher die Schaffung eines neuen Idioms insgesamt. Das ist letztlich die konkrete Anwendung dessen, was Maraldo meint, wenn er Philosophie als die „Über-setzung eines Idioms“ bezeichnet.

\section{Schlussfolgerungen: Von der „Zen-Philosophie“ in Richtung auf „Weltphilosophie“}

Zum Ende dieses Aufsatzes möchte ich zu den Fragen zurückkehren, die ich zu Beginn gestellt habe, und herausarbeiten, was Uedas Typologie zur Frage nach „Zen-Philosophie“ beiträgt. Ich denke, es gibt zwei Arten von Antworten auf diese Frage. Es ist offensichtlich, dass Ueda die Existenz von „Zen-Philosophie“ geltend macht, wie auch die Sinnhaftigkeit, darüber zu reflektieren und $\mathrm{zu}$ reden. Seine Unterscheidung zwischen drei Disziplinen, „Zen“, „Zen-Denken“ und „Philosophie“ veranschaulicht nicht nur die Diversität der Zen-Diskurse, sondern bezeugt außerdem die Sinnlosigkeit der üblichen Einwände gegen die Idee einer Zen-Philosophie, wie im ersten Teil dieses Aufsatzes diskutiert.

\subsection{Uedas Beitrag zu einer „Reflexion der Reflexion“}

Wichtiger noch ist jedoch, dass Uedas mit seinem rhetorisch geschickten Gebrauch der Topoi von „Zen“ und „Welt“ die „Zen-Philosophie“ bzw. das

87 Gross 1993: 215. 
„philosophische Zen“ als eine neue Disziplin herausstellt, die aus der gleichzeitigen „Über-setzung“ des Idioms der akademischen Philosophie wie auch des Idioms des Zen-Buddhismus entsteht. Doch Uedas Modell bringt noch eine weitere, politische, Implikation mit sich. Seine Konzeption einer „Weltphilosophie“ und der „einen Welt“, die an Nishidas „weltliche Welt“ (j. sekaiteki sekai 世界的世界 $)^{88}$ erinnert, macht orientalisierende Dichotomien unmöglich. Denn sie zeigt die Absurdität auf, die einer Dichotomisierung der Welt und einer Essentialisierung von Traditionen und Kulturen wie auch der Praxis eigen ist, eine Disziplin ausschließlich einer regionalen Tradition zuzuordnen im Sinn von „der Westen ist rational“ und „der Osten ist mystisch“.

Ueda vermag es deshalb, die Idee zurückzuweisen, dass „Zen“ und „Westen" unvereinbar seien; vielmehr ist es so, dass Zen-Reden und Philosophie auch in ihrer extremen Form, wie sie sich in den Begegnungen als Dialog in den ZenGenres oder Schriften wie den Analekten (c. Yulu, j. Goroku), den gongan- (Jap.: kōan-)Sammlungen, der Torlose Schranke (c. Wumenguan, j. Mumonkan) und der Niederschrift vom blauen Fels (c. Biyanlu, j. Hekiganroku) übermittelt finden einerseits, in der formal logischen oder dialektischen Philosophie andererseits nicht wesentlich voneinander unterscheiden. Wenn Ueda „die Praxis des UnDenkens“ als die Methode des „Zen“ bestimmt und die „höchste Reflexion“ als die Methode der „Philosophie“, dann impliziert der Einsturz der Dichotomisierung zwischen „Zen“ und „Philosophie“, dass eine klare Unterscheidung zwischen der „Praxis des Un-Denkens“ und „der höchsten Reflexion“ nicht so einfach ist.

Folglich ist ein Dialog zwischen „Zen-Personen“ und „Philosophen“ nicht nur wechselseitig gewinnbringend, sondern auch auch notwendig, um einen Meta-Diskurs der „Reflexion der Reflexion“ einzurichten, der die Grundlage der beiden Disziplinen erhellt, um das Selbstgewahren aller Parteien, die an diesem Dialog beteiligt sind, zu erhöhen, und um eine „Weltphilosophie“ zu schaffen. Praktisch gesehen impliziert dieses Verständnis den Einschluss der Zen-Rede, wenigstens in der Form eines philosophischen Studiums der Zen-Rede, in den philosophischen Diskurs.

Es gibt jedoch einen zweiten, subtileren aber auch bedeutsameren Punkt in Uedas Argumentation. Die Behauptung, „die Praxis des Un-Denkens“ und der „höchsten Reflexion“ seien nicht grundsätzlich verschieden von einem nichtdualistischen Standpunkt mag zwar sinnvoll sein, es handelt es sich aber doch um eine Behauptung mit weitreichenden Implikationen, die deshalb wenigstens einige vorsichtige Erwägungen erfordert. Sie erinnert ganz klar an Nishidas Behauptung, dass Reinheit und Unreinheit „nicht verschieden sind [...]

88 Nishida 1988, Vol. 12: 405-436. 
Verschiedenheit ist immer eine Frage des Grades“, aber was mag das bedeuten? Sogar Ueda insistiert, dass die „Praxis des Un-Denkens“ und die „höchste Reflexion" nicht identisch sind.

\subsection{Dōgens Kritik der Tradition ${ }^{89}$}

Um diese Frage ein wenig weiter $\mathrm{zu}$ untersuchen, werde ich $\mathrm{zu}$ Uedas Modell zurückkehren. Sein Modell gründet auf drei fundamentalen Annahmen: 1) Die Welt, in der wir leben und philosophieren ist ein Ganzes; 2) Ueda weist den metaphysischen Dualismus durchgehend als unhaltbar zurück; 3) Aufgrund ihres nicht-dualen Wesens ist die Welt jedoch ein ineffabile; jeder Ausdruck dieser Erfahrung legt die Struktur von Worten-und-doch-Schweigen frei. Und das ist der Schlüssel zur jetzigen Frage: Worte und Schweigen sind untrennbar ineinander verwoben. Fujita Masakatsu benutzt das Bild einer „Schwellung“ (j. fukurami 膨らみ) $)^{90}$, um seine Überzeugung $\mathrm{zu}$ illustrieren, dass Worte und Schweigen gleichermaßen Bedeutung verdichten.

Diese Haltung ist ziemlich verbreitet unter Denkern der Zen-Tradition. Der prominenteste unter den Zen-Denkern in Japan ist Dōgen. ${ }^{91}$ Er legt den sechsten Fall der Torlosen Schranke neu aus: Traditionell wird auf diesen Text als „Blumen-Predigt“ Bezug genommen und er dient oftmals dazu, zu belegen, dass Schweigen den Worten überlegen sei; genau diesen Punkt bestreitet Dōgen. Der sechste Fall berichtet, dass Shakyamuni Buddha seine Schüler auf dem Geierberg versammelte. Während alle bis auf einen auf seine Predigt warteten, hob er eine Blume auf. In diesem Moment lächelte sein Schüler Mahākāśyapa. Shakyamuni sprach: „Stützt Euch nicht auf Worte, es gibt eine Überlieferung außerhalb der Schriften; ich habe soeben mein Dharma auf Mahākāśyapa übertragen." 92 In Reaktion auf die Haltung, dass der Shakyamuni Buddha auf das fokussierte, was Shibayama Zenkei als „Rede der Nicht-Rede“, die „Überlieferung des Unüberlieferbaren“ und die „geheime Rede“ des „für immer Unnennbaren“93 nennt, bemerkt Dōgen hämisch, dass „hätte Buddha Worte gehasst und das Hochheben von Blumen geliebt, hätte er auch zur späteren Zeit Blumen hochgehoben“94. Dōgen behauptet, dass Shakyamuni Buddha diese

89 Auszüge aus diesem Abschnitt sind veröffentlicht in „When all Dharmas are the Buddhadharma " (Kopf 2015a).

90 Fujita 1998: 106-109.

91 Vgl. dazu auch die umfassende Analyse in Müller 2013: 279-321.

$92 \mathrm{~T}$ 48.2005.293c.

93 Shibayama 1974: 62-64.

94 Dōgen 1969, Vol. 1: 394. 
Worte gebrauchte, um „das Aufsammeln von Blumen“ zu erläutern, wenn er rhetorisch mit Blick auf Shakyamunis Kommentar fragt: „Sind dies Worte oder sind dies keine Worte?"“95.

Ueda übersetzt Dōgens Einsicht in einen philosophischen Diskurs, wenn er feststellt, dass auf der einen Seite das verbale Verständnis einer Erfahrung eine Erfahrung darstellt, während auf der anderen Seite das „Selbstverständnis der Erfahrung“ (j. keiken no jirikai 経験の自理解) „auf Worten beruht“96. Oder in den Worten Fujitas, „Erfahrung und Wörter umfassen zwei Aspekte eines einzelnen Ereignisses“97.

Aber was trägt diese Einsicht zur Diskussion um „Zen-Philosophie“ und „Weltphilosophie“ bei außer den Allgemeinplatz, dass sprachliche Ausdrücke getrennt von Schweigen keine Bedeutung haben und dass, wenn man Wörter im weitesten Sinn von Klängen der Kommunikation unterscheidet, wie es Zhuangzi macht, dann das Gleiche auch umgekehrt gültig ist?

\subsection{Weltphilosophie als lebendiger Diskurs}

Das Verständnis von Worten und Erfahrung als in ihrem Wesen verschieden und sich gegenseitig ausschließend impliziert eine Konzeption der „toten Wörter“, welche definiert und theoretisiert werden, ohne jeden Bezug auf das, was Ueda eine „konkrete Erfahrung“ nennt. Auf der anderen Seite ist eine „konkrete Erfahrung“ frei von Reflexion in Ermangelung von Selbstgewahren. Wenn Erfahrung ein ineffabile ist, so Ueda, dann ist es bedeutungsleer. Es sind die von konkreter Erfahrung erfüllten „lebendigen Wörter“98, die eine Art dritten Terminus darstellen und sich weder in Abstraktionen verlieren noch in der Unerreichbarkeit des Schweigens; auf diese Weise können diese „lebendigen Wörter“ ein Selbstgewahren, das auf Erfahrung basiert und durch Reflexion entwickelt wurde, und folglich eine „Zen-Philosophie“ und „Weltphilosophie“ im Sinne Nishitanis und Uedas bieten.

Worte werden, wenn sie essentialisiert werden, zu toten Objekten der Untersuchung und Theorien werden, in den Worten des Jacques Derrida, logozentrisch; nur im aktiven Engagement, in der Befragung und Transformation können Worte, Theorien und Philosophie als solche den ewig-wechselnden Welten der Erfahrung gerecht werden und folglich Selbstgewahren erzeugen. Wenn dies der

95 Dōgen 1969, Vol. 1: 394.

96 Ueda 1997: 5.

97 Fujita 1998: 124.

98 Ich borge diesen Ausdruck von Jin Y. Park (Park 2008: 131). 
Fall ist, dann sind „Praxis des Un-Denkens“ und die „höchste Reflexion“ zwar nicht identisch, aber unlösbar miteinander verbunden. Es ist eben dieser Akt der Unterscheidung dieser Modalitäten und der Gebrauch dieser Zuschreibungen, der das Bewusstsein des Prozesses des Verstehens und letztlich des Selbstgewahrens vertieft. Es ist die Reifizierung der Kategorien, die zu einer Objektivation und zum Logozentrismus führen. Eine „Weltphilosophie“ ist hingegen offen für eine regenerative Dialektik der „konkreten Erfahrung“ und der systematischen Reflexion.

\section{Abkürzungen}

T: Taisho Taizōkyō. Hrsg. von Junjirō Takakusu und Kaigyoku Watanabe (1961): Tokyo: Taishō Shinshū Daizōkyō Kankōkai.

\section{Bibliographie}

Abe, Masao (1985): Zen and Western Thought. Hrsg. von William R. LaFleur. Honolulu, HI: University of Hawai'i Press.

Abe, Masao (2003): Zen and the Modern World. Hrsg. von Steven Heine. Honolulu, HI: University of Hawaii Press.

Appadurai, Arjun (1996): Modernity at Large: Cultural Dimension of Globalization. Minneapolis, MN: University of Minnesota Press.

Appiah, Kwame Anthony (2005): The Ethics of Identity. Princeton, NJ: Princeton University Press. Dōgen (1969-1970): Dōgen zenji zenshū. Hrsg. von Dōshū Ōkubo. 2 Vols. Tokyo: Chikuma Shobō.

Döll, Steffen (2011): „Ueda Shizuteru's Phenomenology of Self and World: Critical Dialogues with Descartes, Heidegger, and Merleau-Ponty." In: Japanese and Continental Philosophy: Conversations with the Kyoto School. Hrsg. von Bret Davis et al. Indianapolis: Indiana University Press, 120-137.

Fujita, Masakatsu (1998): Gendai shisō toshite no nisihda kitarō (Nishida Kitarō as a Contemporary Thinker). Tokyo: Kōdansha.

Gross, Rita (1993): Buddhism after Patriarchy. Albany, NY: SUNY Press.

Hisamatsu Shin'ichi (1969): Tōyōteki mu: hisamatsu shin'ichi chosakshū 1 (Oriental Nothingness: The Collected Works of Hisamatsu Shin'ichi 1). Tokyo: Risōsha.

Kasulis, Thomas P. (1990): „Does East Asian Buddhism Have an Ethical System?“. Zen Buddhism Today: Annual report of the Kyoto Zen Symposium 8: 41-60.

Kopf, Gereon (2008): „The Self-Contradictory What?--Reflections on how to Teach the Philosophy of Nishida Kitarō". In: Teaching Texts and Contexts: The Art of Infusing Asian Philosophies and Religions. Hrsg. von David Jones und Ellen Klein. Albany: SUNY Press, 129-148.

Kopf, Gereon (2015a): „When all Dharmas are the Buddha-dharma.“ In: Dōgen and Sōtō Zen. Hrsg. von Steven Heine. Oxford: University of Oxford Press, 138-164. 
Kopf, Gereon (2015b): „Towards a Conception of Philosophy as Expression: Approaching Intercultural Philosophy from a Zen Buddhist paradigm.“ In: What is Intercultural Philosophy? Hrsg. von William Sweet. Washington, DC: Council for Research in Values and Philosophy.

Maraldo, John (1995): „Tradition, Textuality, and Trans-lation: The Case of Japan“. In: Japan in Traditional and Postmodern Perspectives. Hrsg. von Charles Fu und Steven Heine. Albany, NY: SUNY, 225-244.

Müller, Ralf (2009): „Watsuji’s Reading of Dōgen's Shōbōgenzō“. In: Confluences \& Cross-Currents. Hrsg. von Raquel Bouso und James W. Heisig. Nagoya: Nanzan, 109-125.

Müller, Ralf (2013): Dōgens Sprachdenken. Historische und symboltheoretische Perspektiven. Freiburg/München: Alber-Verlag.

Müller, Ralf (2016): Nishitanis Vorlesungen zum Shōbōgenzō. Diskussion und Übersetzung. [In Vorbereitung.]

Nishida Kitarō (1988): Nishida kitarō zenshū (The Collected Works of Nishida Kitarō). 20 Vols. Tokyo: Iwanami Shoten.

Nishitani, Keiji (1993): Nishitani keiji chosakushū (Collected Works of Nishitani Keiji). 26 Vols. Tokyo: Iwanami Sōbunsha bunsha, 1986-1995.

Park, Jin (2008): Buddhism and the Politics of Postmodernity: Zen, Huayan, and the Possibility of Buddhist-Postmodern Ethics. New York: Lexington Books.

Sharf, Robert H. (1994): „Whose Zen? Zen Nationalism Revisisted“. In: Rude Aweakenings: Zen, the Kyoto School, and the Question of Nationalism. Hrsg. von James W. Heisig und John C. Maraldo. Honolulu, HI: Hawaii Press, 40-51.

Shibayama, Zenkei (1974): Zen Comments on the Mumonkan. Übers. von Sumiko Kudo. New York: Mentor Book.

Schluetter, Morten (2010): How Zen became Zen: The Dispute over Enlightenment and the Formation of Chan Buddhism in Song-Dynasty China. Honolulu, HI: University of Hawai'i Press

Stauffer, Lee (1989): „Is an Ethical Theory Possible Within Zen Buddhism?“. Southwest Philosophical Studies 11: 80-84.

Suzuki, D. T. (1964): An Introduction to Zen Buddhism. New York: Grove Press.

Suzuki Daisetsu (1968): Suzuki daisetsu zenshū dai 5 kan (The Collected Works of Daisetsu Suzuki, Vol. 5). Tokyo: Iwanami Shoten.

Suzuki Daisetsu (2000): Suzuki daisetsu zenshū dai 13 kan (The Collected Works of Daisetsu Suzuki, Vol.13). Tokyo: Iwanami Shoten.

Takahashi, Satomi (1973): Takahashi satomi zenshū (The Collected Works of Takahashi Satomi). 7 Vol. Tokyo: Fukumura Shuppan.

Ueda, Shizuteru (1997): Kotoba no jitsuzon: zen to bungaku (Words and Reality: Zen and Culture). Tokyo: Chikuma Shobō.

Ueda, Shizuteru (2002a): Ueda shizuteru shū dai 5 kan (Collected Works of Ueda Shizuteru Vol. 5). Tokyo: Iwanami Shoten.

Ueda, Shizuteru (2002b): Nishida Kitarō wa dare ka (Who is Nishida Kitarō?). Tokyo: Iwanami Shoten. 